\title{
К дискуссии о природе селективности сильных ионитов (Ответ на комментарий В.А. Шапошника, опубликованный в журнале «Сорбционные и хроматографические процессы», №2, 2021)
}

\author{
(C) 2021 Долгоносов А.M. \\ Институт геохимии и аналитической химии (ГЕОХИ) РАН, Москва \\ Поступила в редакцию 16.04.2021 г.
}

DOI: $10.17308 /$ sorpchrom.2021.21/3646

В статье продолжена дискуссия по поводу теории селективности сильных ионообменников, предложенной профессором В.А. Шапошником. Применение моделей, использующих дробные заряды, лишено физического смысла. Физически добротная теория должна оперировать целочисленными величинами по отношению элементарному заряду, а волатильность значений энергии кулоновского взаимодействия может быть объяснена различным расстоянием между зарядами, эффектами экранирования и т.П.

В статье приведены математические выводы, касающиеся транспорта ионов разбавленного раствора под действием слабого электрического поля. Эти выводы опираются на закон Нернста-Эйнштейна, связывающий скорость ионов с их диффузионными коэффициентами и температурой.

Согласно известной модели перескоков противоионов между фиксированными зарядами при движении в фазе ионита коэффициенты внутренней диффузии с хорошей точностью подчиняются правилу, выражающему их через коэффициенты распределения и коэффициенты диффузии ионов в жидкости. Последние сильно зависят от температуры, что необходимо учитывать в расчетах. Коэффициенты распределения связаны с избыточной энергией Гиббса при сорбции, соответствующей энергии активации перескока.

Проведено сравнение данных, полученных автором и В.А. Шапошником, по оценке вклада в энергию сорбции кулоновского взаимодействия между фиксированными зарядами ионообменников и противоионами, показывающее (по Китайгородскому) эффективность подхода автора по рассматриваемому вопросу.

Ключевые слова: ионный обмен, внутренняя диффузия, энергия активации переноса противоиона, закон Нернста-Эйнштейна.

Владимир Алексеевич Шапошник в своей реплике [1] не согласился со мной по ряду вопросов, что я объясняю недостаточно подробным моим предыдущим комментарием [2] к его статье [3]. Постараюсь восполнить пробелы и ответить на вопросы глубокоуважаемого оппонента, с которыми он продолжил дискуссию.

1. Введение эффективных нецелых зарядов - это отрицательное качество теории, так как известен непреложный факт о дискретности электрических зарядов. Защищать модели, использующие дробные заряды, не имеет физического смысла. Физически добротная теория должна оперировать целочисленными по отношению к элементарному заряду величинами, а волатильность значений энергии кулоновского взаимодействия 
может быть объяснена различным расстоянием между зарядами, эффектами экранирования и т.п. Имея в виду эти убеждения, мне, очевидно, нет необходимости доказывать свое критическое отношение к результатам вычисления межмолекулярных взаимодействий полуэмпирическими методами квантовой химии, изобилующих множеством параметров, лишенных физического смысла.

2. Мои математические выводы касаются транспорта ионов сильно разбавленного раствора под действием слабого электрического поля и опираются на закон Нернста - Эйнштейна (вариант уравнения Эйнштейна - Смолуховского, выведенного для броуновского движения) пропорциональность скорости фронта ионов их диффузионным коэффициентам, деленным на температуру. Закон Эйнштейна, как и закон Ома, следует строго из флуктуационно-диссипационной теоремы статистической механики. В простых системах, рассмотренных в работе [3], нет никаких причин для нарушения этого фундаментального закона. В статье [4] приведено интересное исследование о применимости этого закона даже в случае сильных электролитов. Итак, для удельной электропроводности мы используем

$$
\kappa \propto \frac{D}{T} .
$$

3. Внутридиффузионные коэффициенты $D$ с хорошей точностью подчиняются правилу, связывающему их с коэффициентами диффузии ионов в жидкости $D_{l i q}$ и коэффициентами распределения Г (формула $D=\frac{D_{l i q}}{1+\Gamma}$, приведена в моем предыдущем комментарии и была проверена для разных ионитов и ионов, например, в $[5,6])$. Эта формула выводится из модели перескоков молекулы по свободной фазе внутри поры от одной точки поверхности к другой. Предположение о наличии подвижной фазы для такого представления коэффициента диффузии избыточно (вода в порах мембраны не движется).

4. Коэффициенты распределения связаны с избыточной энергией Гиббса при сорбции, выполняющей в данном эксперименте роль энергии активации перескока (с противоположным знаком), формулой:

$$
\Gamma+1=\exp \left(-\frac{\Delta G}{R T}\right)=\exp \left(\frac{E_{a}}{R T}\right)
$$

5. Экспериментальная система представляла собой систему «полимер в растворителе», несмотря на отсутствие внешней воды. Поэтому согласно все той же модели перескоков подвижность противоионов в воде должна учитываться в общей проводимости мембраны.

Собирая выводы п.п. 2-5, получаем приведенное в [2] уравнение для результатов эксперимента по электропроводности мембраны при разных температурах:

$$
\begin{aligned}
& \ln \frac{\kappa_{2}}{\kappa_{1}}=\ln \frac{D_{2}}{D_{1}}-\ln \frac{T_{2}}{T_{1}}=\ln \frac{D_{l i q 2}}{D_{l i q 1}}- \\
& -\ln \frac{T_{2}}{T_{1}}-\ln \frac{\Gamma_{2}+1}{\Gamma_{1}+1}=\ln \frac{\kappa_{l i q 2}}{\kappa_{l i q 1}}+\frac{E_{a}}{R}\left(\frac{1}{T_{1}}-\frac{1}{T_{2}}\right)
\end{aligned}
$$

В моем комментарии [2] вместо формулы (2) из статьи В.А. Шапошника [3] записано:

$$
E_{a}=\frac{R\left(\ln \frac{\kappa_{2}}{\kappa_{1}}-\ln \frac{\kappa_{l i q 2}}{\kappa_{\text {liq1 }}}\right)}{\frac{1}{T_{1}}-\frac{1}{T_{2}}}=E_{a,(2)}-\frac{R \ln \frac{\kappa_{l i q 2}}{\kappa_{l i q 1}}}{\frac{1}{T_{1}}-\frac{1}{T_{2}}} .
$$

Из справочных данных по зависимости удельных электропроводностей ионов в воде от температуры следует, что отрицательная поправка к величине, полученной в статье В.А. Шапошника $E_{a,(2)}$, составляет примерно 14.0 кДж/моль. В таблице приведены расчеты, указывающие на то, что итоговая величина, полученная после вычитания этой величины из табличных величин статьи [3], хорошо объясняется доминированием кулоновского члена с традиционно понимаемыми целочисленными зарядами. Точность моих оценок, приведенных в первом комментарии [2], можно улучшить, снизив 
Таблица. Оценка вклада в энергию сорбции кулоновского взаимодействия между фиксированными зарядами ионообменников и противоионами

Table. The estimation of the contribution of the Coulomb interaction between fixed charges of ion exchangers and counterions to the sorption energy

\begin{tabular}{|c|c|c|c|c|c|}
\hline $\begin{array}{c}\text { Проти- } \\
\text { воион }\end{array}$ & Заряд z & $\begin{array}{c}{[3]:} \\
E_{a,(2)}, \\
\text { кДж/моль }\end{array}$ & $\begin{array}{c}{[2]:} \\
E_{a}=E_{a(2)}-14.0, \\
\text { кДж/моль }\end{array}$ & $\begin{array}{c}E_{a} / z, \\
\text { кДж/моль }\end{array}$ & $\begin{array}{c}\text { Потенциал } \\
\text { (кДж/моль) } \\
\text { функцион. } \\
\text { группы }\end{array}$ \\
\hline $\mathrm{Na}^{+}$ & 1 & 21.7 & 7.7 & 7.7 & \multirow[t]{3}{*}{ СГ: 7.5} \\
\hline $\mathrm{Ca}^{2+}$ & 2 & 27.2 & 13.2 & 6.6 & \\
\hline $\mathrm{Al}^{3+}$ & 3 & 38.4 & 24.4 & 8.1 & \\
\hline $\mathrm{Cl}^{-}$ & 1 & 20.4 & 6.4 & 6.4 & \multirow[t]{3}{*}{ ЧАО: 5.5} \\
\hline $\mathrm{SO}_{4}{ }^{2-}$ & 2 & 24.6 & 10.6 & 5.3 & \\
\hline $\mathrm{PO}_{4}^{3-}$ & 3 & 28.8 & 14.8 & 4.9 & \\
\hline
\end{tabular}

СГ - сульфогруппа, ЧАО - четвертичное аммониевое основание.

$\mathrm{SG}$ - sulphogroup, QAB - quaternary ammonium base.

отклонение до 0.6-0.7 кДж/моль от средних значений 7.5 кДж/моль на единицу заряда для катионита и 5.5 кДж/моль для анионита, если учесть, что радиус сульфогруппы на четверть меньше радиуса аммониевого основания (в гидратированном виде).

Высказывание Китайгородского, приведенное Владимиром Алексеевичем в [1], явно должно приветствовать эвристический минимализм моего подхода!

Выступая на семинаре в ИФХЭ РАН в феврале этого года с прояснением своей позиции по данному вопросу, я предложил эксперимент, определяющий какую

\section{Список литературы}

1.Шапошник В.А. // Сорбичионные $u$ хроматографические прочессы. 2021. T.21. № 2. C.285-290.

2.Хамизов Р.Х, Долгоносов А.М. // Сорбиионные и хроматографические проиессы. 2020. Т.20. № 5. С.641-646.

3. Шапошник В.А. // Сорбичонные $u$ хроматографические прочессы. 2020. T.20. № 1. C.48-53. роль играют водородные связи в ионообменной сорбции: активную (член в энергии взаимодействия противоиона с ионообменной группой - точка зрения B.А. Шапошника) или пассивную роль (возможное добавление молекулы растворителя между взаимодействующими ионами - моя точка зрения). Если при добавлении к воде низкомолекулярного спирта константы обмена растут - то водородная связь играет пассивную роль, если падают - то активную. Имеющиеся в литературе данные по сильным ионообменникам свидетельствуют о росте селективности [7].

4. France-Lanord A., Grossman J.C. // Phys. Rev. Lett. 2019. Vol. 122. pp. 136001-1-6

5. Долгоносов А.М., Гарбар А.М. // Журн.физ.химии. 1986. Т.60. № 1. С. 199-200. 6. Долгоносов А.М., Сенявин М.М. и Волощик И.Н., Ионный обмен и ионная хроматография. М. Наука. 1993. С. 28-31. 7. Риман В., Уолтон Г., Ионообменная хроматография в аналитической химии. М. Мир. 1973. С.203. 


\title{
Focus on a discussion about the nature of selectivity of strong ion exchangers (A response to the commentary by V. A. Shaposhnik published in the journal "Sorption and Chromatographic Processes", No 2, 2021)
}

\author{
(C) 2021 Dolgonosov A.M.
}

\author{
Institute of Geochemistry and Analytical Chemistry of the Russian Academy of Sciences, Moscow
}

\begin{abstract}
The article continues the discussion about the theory of selectivity of strong ion exchangers proposed by Professor V. A. Shaposhnik. The application of models using fractional charges lacks any physical meaning. A high quality physical theory should operate with integer values in relation to the elementary charge and the volatility of the values of the energy of Coulomb interaction can be explained by different distances between charges, screening effects, etc.

The article presents mathematical conclusions regarding the transport of ions in a dilute solution under the influence of a weak electric field. These conclusions are based on the Nernst-Einstein law which relates the speed of ions to their diffusion coefficients and temperature. According to the well-known model of counterion jumps between fixed charges during the motion in the ion exchanger phase the internal diffusion coefficients follow the rule which expresses them in terms of distribution coefficients and coefficients of ion diffusion in a liquid with good accuracy. The latter strongly depend on temperature, which must be taken into account in the calculations. The distribution coefficients are related to the excess Gibbs energy during sorption, which corresponds to the energy of the jump activation.

We compared the data obtained by us and V. A. Shaposhnik regarding the estimation of the contribution of the Coulomb interaction between fixed charges of ion exchangers and counterions to the sorption energy. The results showed (according to Kitaygorodsky) the effectiveness of the author's approach to the considered issue.
\end{abstract}

Keywords: ion exchange, internal diffusion, the activation energy of counterion transfer, Nernst Einstein law.

\section{References}

1. Shaposhnik V.A., Sorbtsionnye i khromatograficheskie protsessy, 2021, Vol. 21, No 2, pp. 285-290.

2. Khamizov R.H, Dolgonosov A.M., Sorbtsionnye i khromatograficheskie protsessy. 2020, Vol. 20, No 5, pp. 641-646.

3. Shaposhnik V.A., Sorbtsionnye i khromatograficheskie protsessy. 2020, Vol. 20, No 1, pp. 48-53.

Долгоносов Анатолий Михайлович - д.х.н., вед. научный сотрудник, лаб. сорбционных методов, ГЕОХИ РАН, Москва
4. France-Lanord A., Grossman J.C., Phys. Rev. Lett., 2019, Vol. 122, pp.136001-1-6

5. Dolgonosov A.M., Garbar A.M., ZHurn.fiz.himii, 1986, Vol. 60, No 1, pp.199-200.

6. Dolgonosov A.M., Senyavin M.M. i Voloshchik I.N., Ionnyj obmen i ionnaya hromatografiya. M., Nauka, 1993, pp. 28-31.

7. Riman V., Uolton G., Ionoobmennaya hromatografiya $\mathrm{v}$ analiticheskoj himii. M., Mir, 1973, pp. 203

Dolgonosov Anatoly M. - Dr. Sci. (Chem.), Lead. research sci., Lab. of sorption methods, GEOKHI RAS, Moscow, e-mail: amdolgo@mail.ru 